Original Paper

http://indexmedicus.afro.who.int

\title{
Pêche et paramètres de reproduction de Pellonula leonensis Boulenger 1916, dans le lac de Taabo (Fleuve Bandama, Côte d'Ivoire): implications pour une exploitation durable du stock
}

\author{
Lucien Ayémin Boutonner KRAIDY ${ }^{1}$, Naminata KONE ${ }^{1 *}$, Siaka BERTE ${ }^{2}$, \\ Gervais N'ZI KONAN ${ }^{2}$, Stanislas Sylvain YAO $^{2}$ et Paul Essétchi KOUAMELAN ${ }^{2}$ \\ ${ }^{I}$ Département de Sciences et Technologie, Ecole Normale Supérieure (ENS) d'Abidjan, \\ 08 BP 10 Abidjan 08, Côte d'Ivoire. \\ ${ }^{2}$ Laboratoire d'Hydrobiologie, UFR Biosciences, Université Félix Houphouët-Boigny, \\ 22 BP 582 Abidjan 22, Côte d'Ivoire. \\ Auteur correspondant ; E-mail : k_naminata@yahoo.fr; Tél. : (+225) 05764744
}

\section{RESUME}

La pêche et la reproduction de Pellonula leonensis provenant du lac de barrage de Taabo, ont été étudiées de novembre 2008 à octobre 2009, en vue d'une meilleure gestion du stock de l'espèce. L'engin de pêche utilisé pour la capture de $P$. leonensis est la senne de plage de maille variant entre 6 et $8 \mathrm{~mm}$. L'effort de pêche fourni est de 3547 sorties de pêche par an. La prise par unité d'effort est de $59,52 \mathrm{~kg} / \mathrm{sortie}$ de pêche. La production totale de $P$. leonensis à Taabo-village, a été estimée à 211,860 tonnes. Au total, 621 spécimens de $P$. leonensis (de longueur standard comprise entre 27 et $65 \mathrm{~mm}$ pour une moyenne de 40,33 $\pm 6,08 \mathrm{~mm}$ et de masse variant de 0,078 à $5,54 \mathrm{~g}$ avec une moyenne de $1,19 \pm 0,63 \mathrm{~g}$ ) ont été examinés pour l'étude de la reproduction. Le sex-ratio de $1: 0,68$ est en faveur des mâles. La taille de première maturité sexuelle est de $27,45 \mathrm{~mm}$ et de $27,48 \mathrm{~mm}$ respectivement chez les femelles et les mâles. Les évolutions du rapport gonadosomatique et des fréquences des stades matures ont révélé que la période de reproduction de $P$. leonensis s'étend principalement de décembre à avril et accessoirement en juin et août.

(C) 2014 International Formulae Group. All rights reserved.

Mots-clés : Effort de pêche, production annuelle, taille de première maturité, période de reproduction, Côte d'Ivoire.

\section{INTRODUCTION}

La pêche est l'une des activités qui contribue de façon déterminante au bien-être et à la prospérité des hommes. De plus, les produits de la pêche font partie des denrées alimentaires de base les plus échangées dans le monde (FAO, 2012). Le poisson représente la principale source de protéines (plus de $70 \%$ ) pour les populations africaines (Vanga, 2011). Selon la FAO (2012), promouvoir la pêche durable peut encourager une gestion plus avisée de l'écosystème dans son ensemble.

Toutefois, si tel n'est pas le cas, les ressources peuvent chuter brutalement. L'un des effets les plus marqués de la pêche se manifeste sur le plan de la démographie, par la réduction de la taille moyenne des espèces et la disparition des individus de grande taille. En effet, si la pêcherie débute en général avec 
des engins à grandes mailles, la taille de celles-ci diminue au fur et à mesure que les captures de grands individus se font plus rares (Lévêque et Paugy, 2006).

En Côte d'Ivoire, la pêche continentale repose sur un ensemble de pêcheries lacustres dont les principales sont les lacs de Kossou, Buyo, Ayamé I et Taabo (Da Costa et Dietoa, 2007). Dans le lac de Taabo, l'espèce Pellonula leonensis Boulenger, 1916, fait l'objet d'une exploitation importante avec une production de 20 tonnes par an et par unité de pêche (Yao, 2008). L'on observe cependant, une différence notable de taille entre les spécimens de $P$. leonensis capturés dans le lac de Taabo et ceux provenant des lacs de Kossou et de Faé. Les individus du lac de Taabo ont une taille bien plus petite que ceux des deux autres lacs (Yao, 2008).

Du point de vue de la gestion et/ou de la conservation, une connaissance approfondie de la biologie de la reproduction d'une espèce permet de mieux gérer le stock de géniteurs et partant, de la population. Ainsi, déterminer la taille de première maturité sexuelle permet de suggérer une taille minimale de capture dans les pêcheries (Mehanna, 2007). De même, la connaissance de la biologie de la reproduction d'une espèce permet de déterminer les dates d'interdiction de sa pêche ou de mise en reserve de sa frayère (Konan, 2010).

En conséquence, le présent travail, après une présentation de la pêche de l'espèce dans le lac de Taabo et de l'analyse de ses paramètres de reproduction, propose une approche de gestion rationnelle fondée sur la protection des stocks de géniteurs en période de reproduction.

\section{MATERIEL ET METHODES Milieu d'étude}

Le barrage hydroélectrique de Taabo créé en 1978 est implanté sur le cours principal du fleuve Bandama (Figure 1) à environ $110 \mathrm{~km}$ en aval du confluent du
Bandama blanc et du Bandama rouge et à environ $120 \mathrm{~km}$ en aval du barrage de Kossou (Kouassi et al., 2007). Le lac de Taabo issu de ce barrage est situé entre $06^{\circ} 20^{\prime}$ et $06^{\circ} 40^{\prime}$ de latitude nord et $5^{\circ}$ et $5^{\circ} 30^{\prime}$ longitude ouest. Avec un volume de $625.10^{6} \mathrm{~m}^{3}$, cette retenue d'eau couvre une superficie de $69 \mathrm{~km}^{2}$. Ce lac draine un bassin versant de $58700 \mathrm{~km}^{2}$ et son débit moyen annuel est de $128,70 \mathrm{~m}^{3} / \mathrm{s}$ (Kouassi et al., 2007 ; Aliko et al., 2010). Le lac de Taabo est sous l'influence du climat attiéen avec un régime hydrologique de type équatorial de transition (Savané et Konaré, 2010).

\section{Collecte des données}

Des enquêtes sur les débarquements de Pellonula leonensis ont été menées quotidiennement de novembre 2008 à octobre 2009. Pendant cette même période, des échantillons de ce poisson ont été collectés à partir de la pêche commerciale pour l'étude des paramètres de reproduction. Les pêcheurs de Taabo-village utilisent principalement des sennes de plage, de mailles variant entre 6 et 8 $\mathrm{mm}$ pour la pêche de $P$. leonensis. La pêche s'effectue en équipe composée de 3 à 4 personnes. Vingt (20) équipes possédant chacune une senne ont été enregistrées.

\section{Etude de la pêche}

L'effort de pêche considéré dans la présente étude est l'ensemble des sorties de pêche, l'engin de pêche utilisé étant la senne de rivage. Il est exprimé en nombre de sorties de pêche. L'unité d'effort retenue est la sortie de pêche. La prise par unité d'effort (PUE) est la masse des captures réalisées par un pêcheur durant une sortie de pêche. Elle est exprimée en $\mathrm{kg} / \mathrm{sortie}$ de pêche. Pour chaque mois, une prise par unité moyenne $\left(\mathrm{PUE}_{\mathrm{m}}\right)$ a été déterminée à partir de l'ensemble des captures journalières enregistrées durant le mois considéré selon la formule suivante : 
$\mathrm{PUE}_{\mathrm{m}}=\mathrm{P}_{\mathrm{t}} / \mathrm{N}_{\mathrm{t}}$ avec $\mathrm{PUE}_{\mathrm{m}}=$ prise moyenne mensuelle par unité d'effort; $P_{t}=$ masse totale en $\mathrm{kg}$ des captures journalières débarquées par l'ensemble des pêcheurs enquêtés dans le mois et $\mathrm{N}_{\mathrm{t}}=$ nombre total de sorties de pêche dans le mois.

Les captures mensuelles $\left(\mathrm{C}_{\mathrm{i}}\right)$ sont estimées par la sommation des captures journalières réalisées pendant le mois $\mathrm{i}$. La capture totale annuelle $\left(\mathrm{C}_{\mathrm{a}}\right)$ ou production annuelle est obtenue par la sommation des captures totales mensuelles.

\section{Détermination des paramètres de la reproduction}

Les spécimens échantillonnés sont individuellement mesurés au millimètre près à l'aide d'un ichtyomètre millimétré, pesés au millième de gramme près avec une balance électronique de type SARTORIUS model BP $310 \mathrm{~S}$ de précision $0,001 \mathrm{~g}$ et disséqués en vue de déterminer le sexe et le stade de maturité sexuelle. Les gonades et le foie sont ensuite prélevés et également pesés ainsi que le poisson éviscéré. Toutes ces mesures ont été effectuées au laboratoire.

Le sex-ratio (SR), correspondant à la proportion relative des individus des deux sexes dans une population à un moment donné, a été déterminé selon la formule suivante: $\mathrm{SR}=\mathrm{M} / \mathrm{F}$ avec $\mathrm{F}=$ nombre de femelles et $\mathrm{M}=$ nombre de mâles.

La formule utilisée pour le calcul du facteur de condition est la suivante:

$\mathrm{Kc}=\frac{\mathrm{P}}{\mathrm{Ls}^{3}} \times 10^{5}$ avec $\mathrm{P}=$ masse du poisson

(g) et $\mathrm{Ls}=$ longueur standard du poisson (mm).

La taille de première maturité sexuelle $\left(\mathrm{Ls}_{50}\right)$ a été définie comme la taille à laquelle $50 \%$ des individus de la population (mâles et des femelles) sont matures (Sylla et al., 2009). Sont considérés comme matures, les poissons présentant des gonades au stade supérieur ou égal à 3. Le pourcentage des individus matures dans chaque classe de taille (intervalle $1 \mathrm{~mm}$ ) a été calculé en fonction du nombre total d'individus pour chaque classe de taille. La taille de première maturité sexuelle a été estimée en ajustant par la fonction logistique d'une régression non linéaire reliant les proportions des individus matures et la longueur standard du poisson. Cette fonction de forme sigmoïde permet de déterminer avec précision la taille de la première maturité sexuelle $\left(\mathrm{Ls}_{50}\right)$.

$\mathrm{La}$ formule s'écrit comme suit : $\mathrm{P}=\frac{\mathrm{e}^{(\alpha+\beta \mathrm{Ls})}}{1+\mathrm{e}^{(\alpha+\beta \mathrm{Ls})}}$ et $\mathrm{Ls}_{50}=\frac{-\alpha}{\beta}$

avec $\mathrm{P}=$ proportion des individus matures ;

$\mathrm{Ls}=$ longueur standard $(\mathrm{mm})$;

$\alpha$ et $\beta=$ constantes.

Les rapports gonado-somatique (RGS) et hépato-somatique (RHS) ont été calculés à partir des formules suivantes :

RGS $=\frac{P_{g}}{P_{\text {év }}} \times 100$ et RHS $=\frac{P_{f}}{P_{\text {év }}} \times 100$ avec $P_{g}=$ masse des gonades $(g) ; P_{f}=$ masse du foie (g) et Pév $=$ masse du poisson éviscéré (g).

Les pourcentages des stades les plus avancés de la maturité des gonades femelles (stades $3 ; 4 ; 5$ et 6 ) et le rapport gonadosomatique moyen (RGS moyen) ont été couplés afin de préciser la période principale de reproduction de l'espèce.

\section{Analyse des données}

Les tailles de première maturité sexuelle ont été comparées en fonction du sexe à l'aide du test $\mathrm{de} \mathrm{Khi}^{2}\left(\chi^{2}\right)$ avec le logiciel Statistica version 7.1. Le test de $\mathrm{Khi}^{2}$ a également été utilisé pour comparer les pourcentages des sexes (sex-ratio) des différents mois et saisons au sex-ratio théorique $(1: 1)$. 


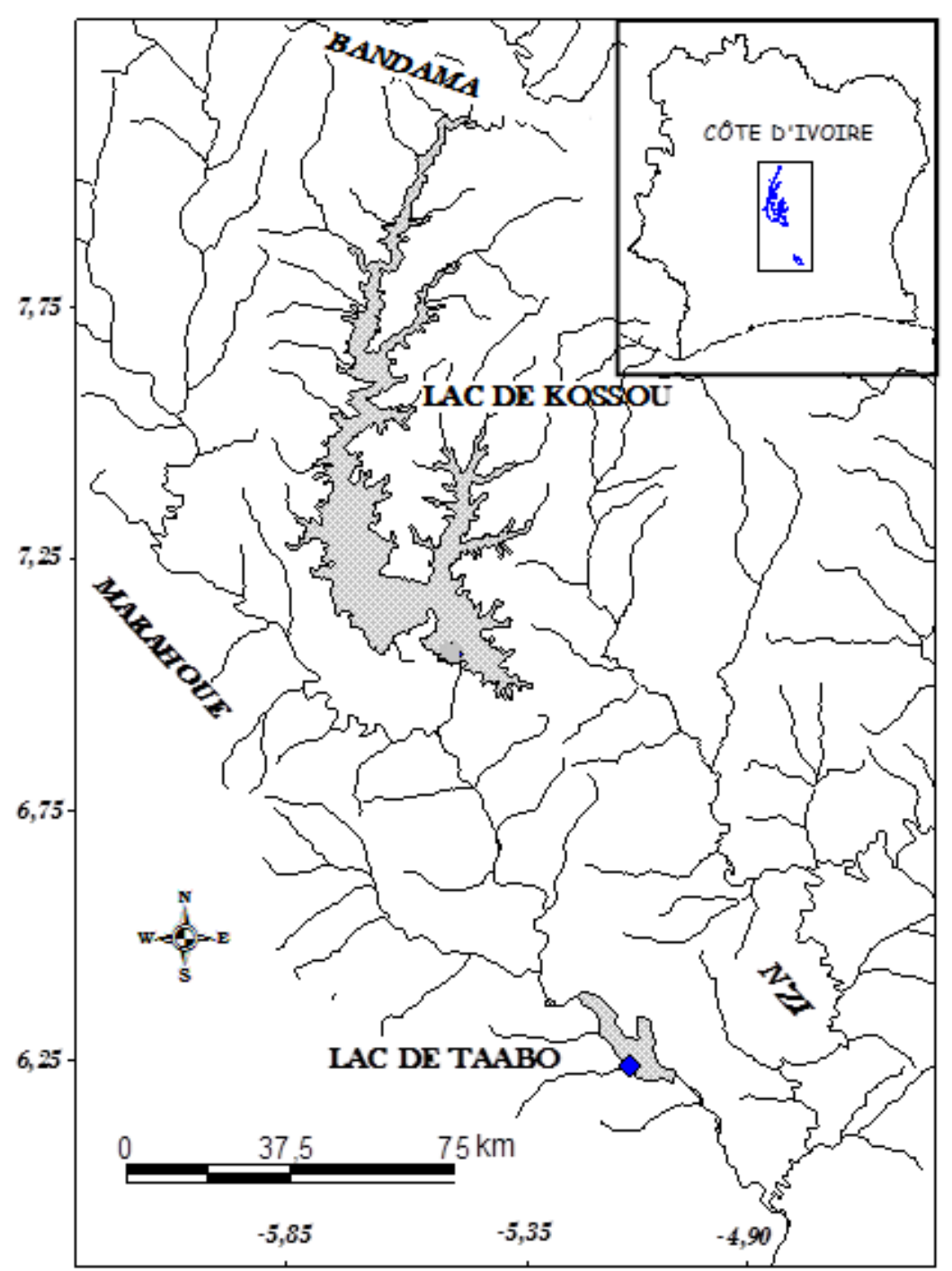

Figure 1 : Localisation de la station d'échantillonnage ( $)$ sur le lac de barrage de Taabo.

\section{RESULTATS}

\section{Effort de pêche}

Au total, 3547 sorties de pêches ont été enregistrées au cours de l'année d'étude avec une moyenne de 295 sorties/mois. L'effort de pêche mensuel évolue en dents de scie avec un pic important en octobre (423 sorties). La valeur la plus basse est observée en janvier avec 246 sorties de pêche (Figure 2).
En moyenne, 9,75 \pm 1,51 sorties de pêches sont notées par jour (Tableau 1). Le plus grand nombre de sorties journalières a été observé en octobre (14 sorties) et le plus faible a été noté en décembre et en janvier ( 8 sorties). Par mois, les pêcheurs effectuent entre 28 et 31 jours de pêche avec une moyenne de $30,33 \pm 0,98$ jours. 


\section{Prise par unité d'effort}

La PUE est de $59,52 \mathrm{~kg} / \mathrm{sortie}$ de pêche. Pour une valeur moyenne de 9,75 \pm 1,51 sorties par jour, la prise journalière équivaut à 580,32 $\pm 89,87 \mathrm{~kg}$ de Pellonula leonensis. $\mathrm{La}$ prise mensuelle par unité d'effort évolue en dents de scie au cours de l'année (Figure 3). Quatre pics sont observés, dont le plus important en septembre $(89,69 \pm$ $40,22 \mathrm{~kg} / \mathrm{sortie}$ ). La valeur la plus faible de la PUE $_{\mathrm{m}}$ est notée en février $(32,76 \pm 20,10$ $\mathrm{kg} /$ sortie).

\section{Production totale}

La production totale de $P$. leonensis déterminée est de 211,860 tonnes. La plus forte production est notée en octobre avec 30,558 tonnes. Le mois de février enregistre le taux de production le plus bas avec 240 tonnes (Tableau 1).

\section{Sex-ratio}

Le sex-ratio obtenu est de 0,68 femelle pour 1 mâle. Il est significativement différent du sex-ratio théorique qui est de $1: 1$. Un sexratio en faveur des femelles a été observé pendant les mois de novembre, février, avril, juin et août. Le sex-ratio est en faveur des mâles au cours des autres mois de l'année d'échantillonnage et pendant les deux saisons (Tableau 2).

\section{Taille de première maturité sexuelle}

Les mâles sont matures à $27,48 \mathrm{~mm}$ et les femelles le sont à 27,45 mm (Figure 4). Aucune différence significative n'existe entre ces deux tailles. Les plus petits individus femelles et mâles matures mesurent $28 \mathrm{~mm}$ (LS).

\section{Rapport hépato-somatique}

Les valeurs du RHS sont maximales en janvier aussi bien chez les femelles que chez les mâles avec respectivement $4,6 \pm 0,92 \%$ et $3,32 \pm 1,49 \%$ Les valeurs minimales sont enregistrées en mai $(0,85 \pm 0,13 \%)$ pour les femelles et en mars $(0,90 \pm 0,35 \%)$ pour les mâles (Figure 5).

\section{Rapport gonado-somatique}

Chez les femelles, l'évolution du RGS présente trois pics d'inégale importance. Le pic le plus élevé est observé en janvier (19,06 $\pm 1,4 \%)$. Vient ensuite celui noté en octobre $(13,62 \pm 4,09 \%)$. Le pic le moins élevé est enregistré en juin avec $9,53 \pm 4,11 \%$ (Figure 5). Une baisse du RGS est observée de janvier à mai où il atteint sa valeur minimale $(5,75 \pm$ $0,2 \%)$. Chez les mâles, les valeurs maximales du RGS sont notées en janvier $(9,01 \pm 2,15 \%)$ et novembre $(7,5 \pm 1,88 \%)$. La valeur la plus faible est notée en juillet $(3,27 \pm 1,04 \%)$.

\section{Facteur de condition}

Chez les femelles, le maximum de ce paramètre est enregistré en mai $(2,01 \pm 0,02)$. Le facteur de condition moyen le plus bas a été noté pendant le mois de janvier $(1,4 \pm$ $0,14)$. Pour les mâles, la moyenne la plus élevée du facteur de condition est observée en novembre $(2,04 \pm 0,13)$. La valeur la plus basse est enregistrée pendant le mois de janvier avec 1,35 $\pm 0,18$ (Figure 5).

\section{Période de reproduction}

Toutes les femelles capturées en janvier sont matures. Les femelles matures pêchées en février et en avril représentent respectivement $52,94 \%$ et $60,41 \%$ de l'ensemble des femelles capturées pendant ces mois. Le rapport gonado-somatique moyen pendant ces mois est compris entre 7,39\% en avril et 19,06\% en janvier. Les mois d'août, octobre et décembre enregistrent des pourcentages de femelles matures compris entre 25 et $47 \%$ et des valeurs de RGS moyen 
comprises entre 4,86 et $8,85 \%$. Les proportions les plus faibles de femelles matures sont observées en mai $(12,50 \%)$, en septembre $(6,2 \%)$ et en juillet où aucune femelle mature n'a été capturée. Les plus faibles valeurs $\mathrm{du}$ RGS moyen sont enregistrées pendant ces mêmes mois. Dans le lac de Taabo, la ponte des oeufs de $P$. leonensis s'effectue principalement de décembre à avril avec un important pic de ponte en janvier et un pic moins important en avril. Elle se poursuit ensuite pendant les mois de juin et août (Figure 6).

\section{Distribution des fréquences de taille des ovocytes}

La distribution des fréquences des diamètres ovocytaires des gonades au stade 2 montre deux groupes d'ovocytes (Figure 7).
Le premier groupe est constitué d'ovocytes de diamètre compris entre 0,01 et $0,03 \mathrm{~mm}$. Le second concerne les ovocytes dont le diamètre varie entre 0,04 et $0,07 \mathrm{~mm}$. Les ovaires aux stades de maturité 3 , 4 et 5 présentent trois groupes d'ovocytes. Le premier groupe est composé d'ovocytes de petite taille avec des diamètres compris entre 0,01 et $0,05 \mathrm{~mm}$. Le second groupe est constitué par les ovocytes de taille moyenne (diamètres situés entre 0,06 et $0,12 \mathrm{~mm}$ ) et le troisième groupe est composé d'ovocytes de taille plus grande (diamètres compris entre 0,13 à 0,45 $\mathrm{mm}$ ). Dans les ovaires au stade 6 , les ovocytes de différentes tailles sont observés. Outre quelques ovocytes de grande taille, les ovocytes du stock de réserve sont observés en grand nombre.

Tableau 1 : Nombres de sorties de pêche par jours, de jours de pêche et production mensuelles de Pellonula leonensis à Taabo-village de novembre 2008 à octobre 2009.

\begin{tabular}{lccc}
\hline Mois & $\begin{array}{c}\text { Nb de jours de } \\
\text { pêche }\end{array}$ & $\begin{array}{c}\text { Nb de sorties de } \\
\text { pêche/jour }\end{array}$ & Productions (kg) \\
\hline Novembre & 30 & 11 & 12672 \\
Décembre & 31 & 8 & 14091 \\
Janvier & 31 & 8 & 21384 \\
Février & 28 & 10 & 9240 \\
Mars & 31 & 10 & 20504 \\
Avril & 29 & 9 & 12364 \\
Mai & 31 & 9 & 14432 \\
Juin & 30 & 10 & 19745 \\
Juillet & 31 & 10 & 17611 \\
Août & 31 & 9 & 15400 \\
Septembre & 30 & 9 & 23859 \\
Octobre & 31 & 14 & 30558 \\
\hline Moyenne/ Total & $30,33 \pm 0,98$ & $9,75 \pm 1,51$ & 211860 \\
\hline \multicolumn{2}{c}{$\mathrm{Nb}=$ nombre } & &
\end{tabular}


Tableau 2 : Longueur standard, nombre de femelles et de mâles et sex-ratio de Pellonula leonensis dans le lac de Taabo.

\begin{tabular}{|c|c|c|c|c|}
\hline \multirow[t]{2}{*}{ Mois } & Femelles & Mâles & Sex-ratio & \multirow[t]{2}{*}{$\chi^{2}$} \\
\hline & $\mathbf{L}_{\min }-\mathbf{L}_{\max }(\mathbf{m m})$ & $\mathbf{L}_{\min }-\mathbf{L}_{\max }(\mathbf{m m})$ & $\mathbf{M}: \mathbf{F}$ & \\
\hline Novembre & $32-65(44)$ & $33-56(19)$ & $1: 2,31$ & $6,73^{*}$ \\
\hline Décembre & $30-53(36)$ & $34-55(60)$ & $1: 0,60$ & 4,03 \\
\hline Janvier & $45-50(5)$ & $38-55(39)$ & $1: 0,12$ & $18,76^{*}$ \\
\hline Février & $35-52(17)$ & $28-48(16)$ & $1: 1,06$ & 0,02 \\
\hline Mars & $31-42(6)$ & $31-55$ (27) & $1: 0,22$ & $9,33^{*}$ \\
\hline Avril & $27-52(48)$ & $27-47(40)$ & $1: 1,20$ & 0,49 \\
\hline Mai & $37-57(16)$ & $35-65(28)$ & $1: 0,57$ & 2,20 \\
\hline Juin & $28-44(23)$ & $32-56(21)$ & $1: 1,09$ & 0,06 \\
\hline Juillet & $38-38(1)$ & $35-48(43)$ & $1: 0,02$ & $29,74^{*}$ \\
\hline Août & $30-48(28)$ & $27-45(16)$ & $1: 1,75$ & 2,20 \\
\hline Septembre & $27-40(16)$ & $27-40(28)$ & $1: 0,57$ & 2,20 \\
\hline Octobre & $28-40(12)$ & $28-47(32)$ & $1: 0,37$ & $6,20^{*}$ \\
\hline TOTAL & 252 & 369 & $1: 0,68$ & $14,75^{*}$ \\
\hline Saison pluvieuse & 144 & 186 & $1: 0,77$ & 3,57 \\
\hline Saison sèche & 108 & 183 & $1: 0,59$ & $12,98^{*}$ \\
\hline
\end{tabular}

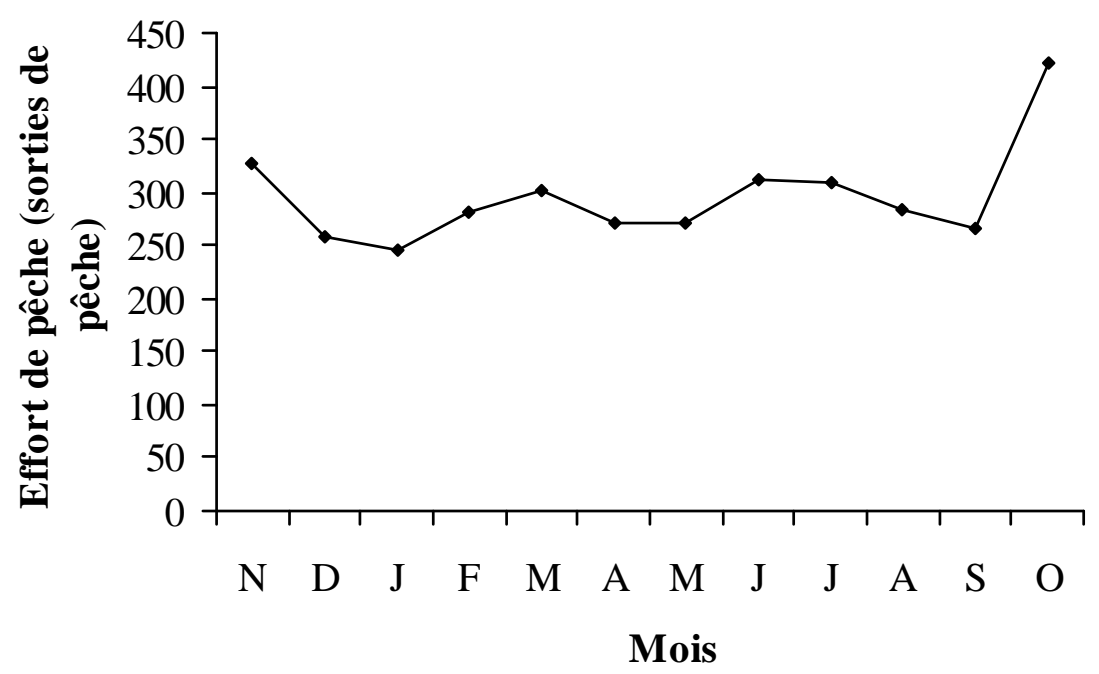

Figure 2 : Variation mensuelle de l'effort de pêche à Taabo-village. 


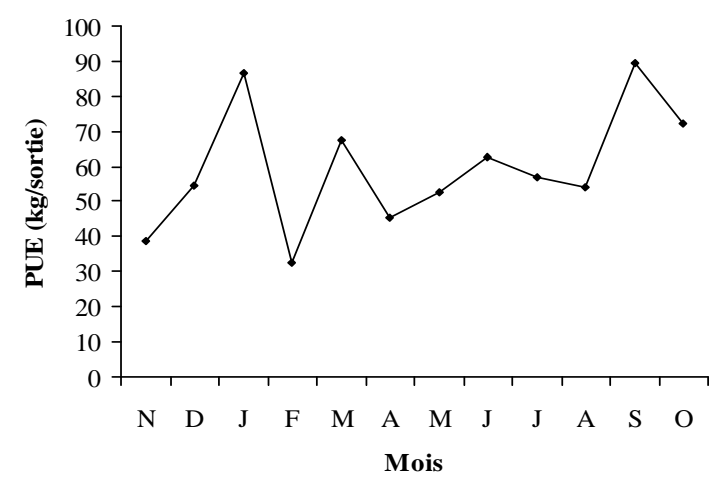

Figure 3 : Variation mensuelle des prises moyennes mensuelles par unité d'effort $\left(\mathrm{PUE}_{\mathrm{m}}\right)$ à Taabovillage.
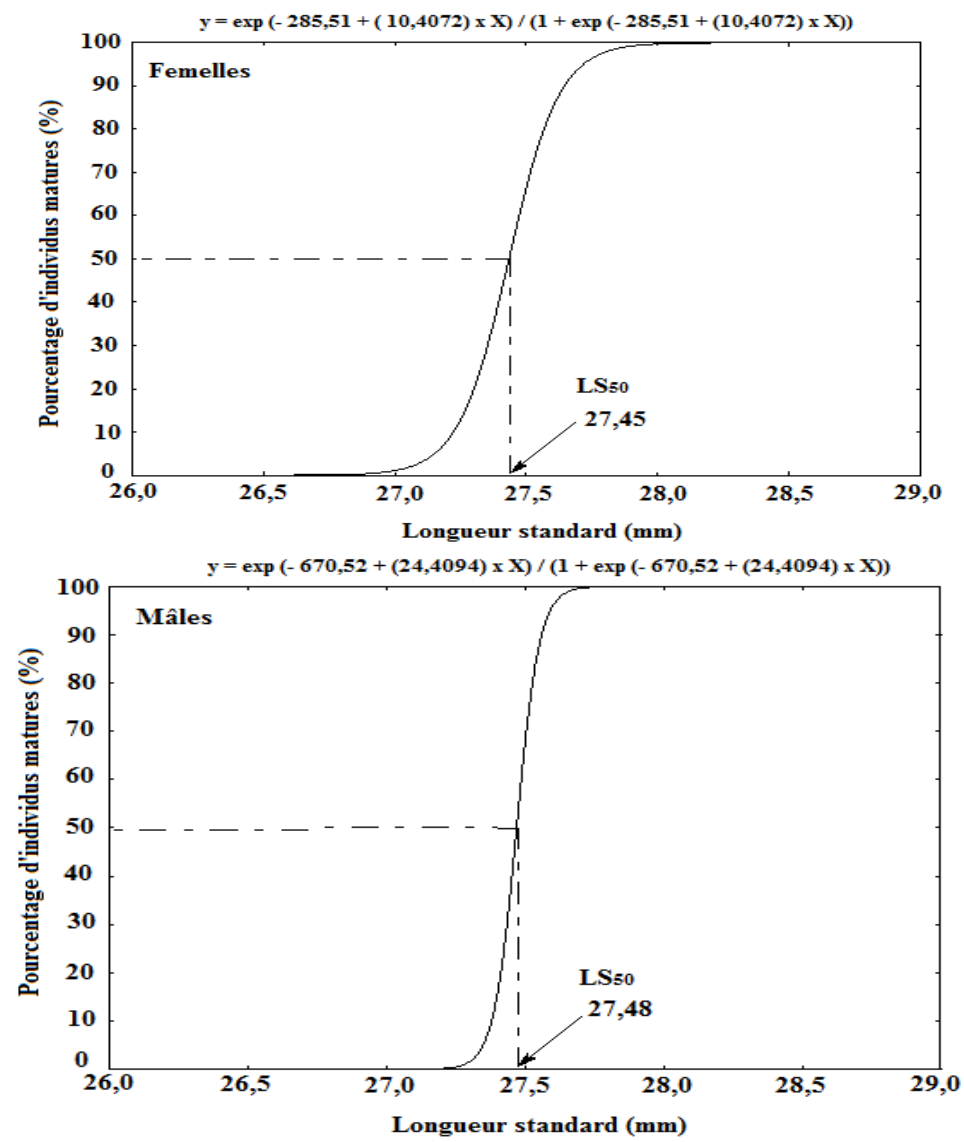

Figure 4: Détermination de la taille de première maturité sexuelle $\left(\mathrm{LS}_{50}\right)$ en fonction du pourcentage des individus femelles et mâles matures de Pellonula leonensis. 

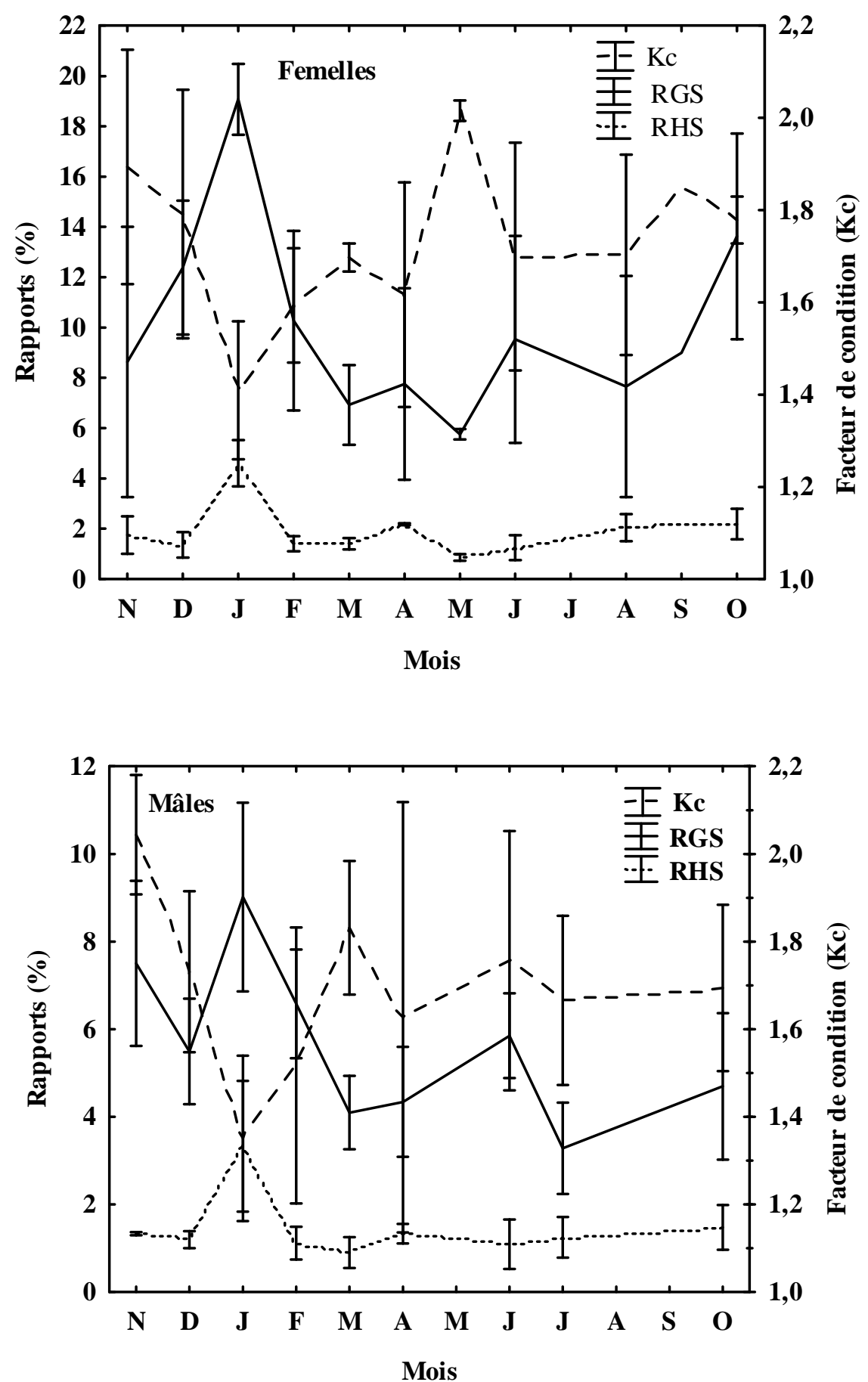

Figure 5 : Variations mensuelles des rapports hépato-somatique, gonado-somatique et du facteur de condition des femelles et mâles matures de Pellonula leonensis dans le lac de Taabo. 


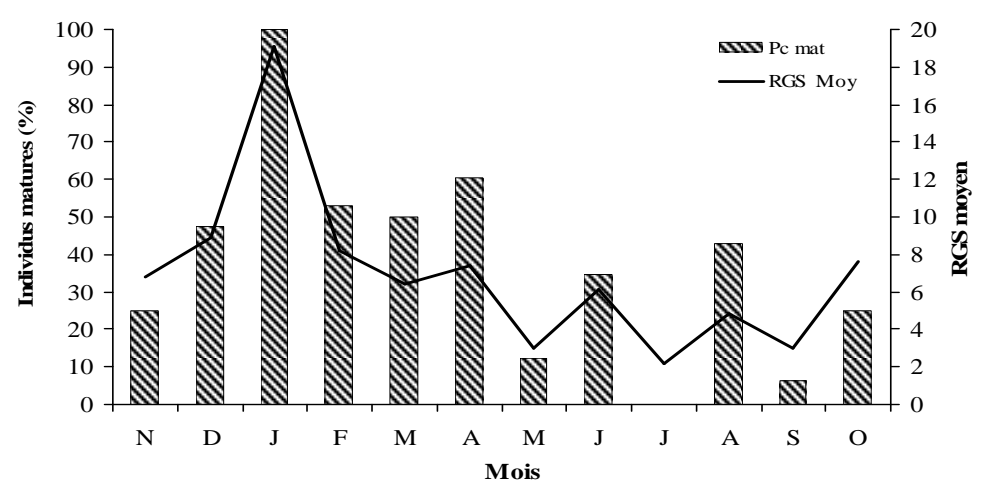

Figure 6: Evolutions mensuelles du rapport gonado-somatique moyen et du pourcentage des femelles matures de Pellonula leonensis dans le lac de Taabo. \% mat = pourcentage d'individus matures ; RGS moy = rapport gonado-somatique moyen.
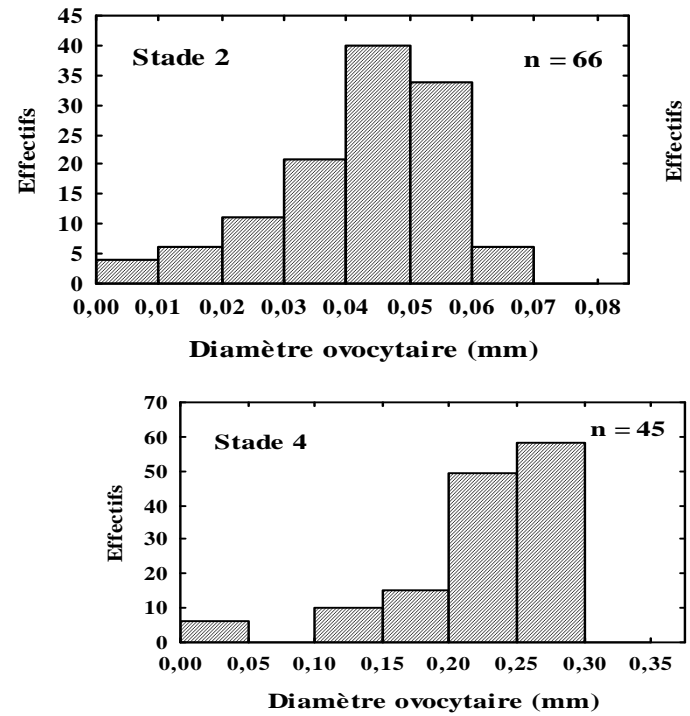
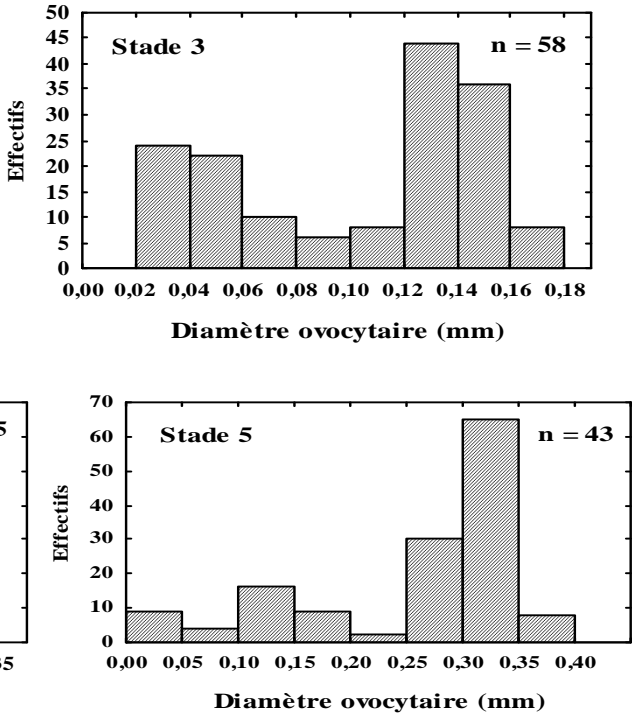

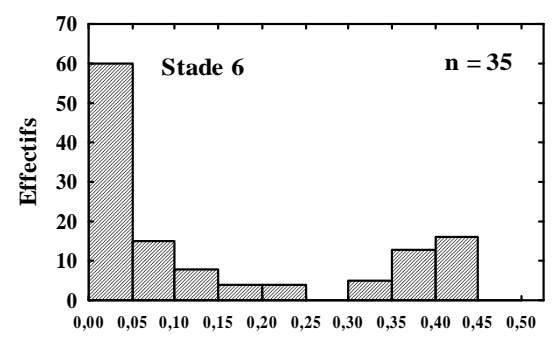

Diamètre ovocytaire $(\mathbf{m m})$

Figure 7 : Distribution des fréquences de taille des ovocytes dans les gonades à différents stades de maturité sexuelle chez Pellonula leonensis dans le lac de Taabo. $\mathrm{n}=$ nombre de femelles. 


\section{DISCUSSION}

Le sex-ratio observé pour l'ensemble des captures de Pellonula leonensis dans le lac de Taabo montre une dominance des mâles (1: 0,68). Kingdom et Allison (2009) ont obtenu des résultats similaires dans la rivière Lower Nun du Delta du Niger chez la même espèce. La variation saisonnière du sex-ratio a montré la prédominance des mâles pendant les deux saisons. La prédominance d'un des sexes est un phénomène relativement fréquent chez de nombreuses espèces de poissons téléostéens (Layachi et al., 2007; Atsé et al., 2009). Selon Santos et al. (2007), les mâles sont prédominants en général, chez les téléostéens, en période de reproduction alors qu'en période de repos sexuel, ce sont les femelles qui prédominent. Selon ChemmamAbdelkader et al. (2007), pendant la période de reproduction, les mâles se concentrent autour des femelles. Le nombre des individus mâles devient alors plus important que celui des femelles, d'où un sex-ratio en faveur des mâles. La sex-ratio général en faveur des mâles observés dans le lac de Taabo serait dû à une période de reproduction très étalée dans le temps.

Les valeurs de la taille de première maturité sexuelle obtenues à Taabo sont de 27,45 mm pour les femelles et 27,48 $\mathrm{mm}$ (LS) pour les mâles. Elles sont inférieures à celles notées dans le lac de Kossou par Koné et al. (2011). Ces auteurs ont en effet rapporté que la taille de première maturité sexuelle est atteinte à $69,42 \mathrm{~mm}$ pour les femelles et à 75,49 mm (LS) pour les mâles. Cette variabilité intraspécifique de la taille de maturité sexuelle a été observée chez le Clupeidae Ethmalosa fimbriata par N'Goran (1995) et chez le Cichlidae Aequidens tetramerus par Silva et al. (2013). Lévêque et al. (2006) attribuent les variations de la taille de première maturité en fonction des bassins ou habitats considérés, au fait que les poissons développent des stratégies en fonction du milieu en vue d'une meilleure adaptation et de tirer profit de leur écosystème. En outre, Paugy (2002) a rapporté que selon la règle générale, plus les rivières sont grandes, plus la taille de la première maturité sexuelle des poissons est élevée. Les activités anthropiques telles que la pêche abusive et l'utilisation de certains engins de pêche expliqueraient les différences de taille de première maturité. En effet, Atsé et al. (2009) ont montré que la surpêche entraine une réduction de la taille de première maturité sexuelle. Dans la présente étude, les différences de taille notées pourraient donc s'expliquer par le fait que la pêche commerciale de $P$. leonensis est très intense à Taabo avec une production annuelle de $211860 \mathrm{~kg}$. En effet, 580,32 $\mathrm{kg}$ en moyenne de $P$. leonensis sont débarqués par jour par les pêcheurs. Ces derniers effectuent en moyenne 30,33 jours de pêche par mois. En outre, les pêcheurs de cette localité utilisent des sennes de rivage avec un maillage compris entre 6 et $8 \mathrm{~mm}$ pour la pêche de ce poisson. Otobo (1978) a observé dans le lac Kainji, des tailles de première maturité sexuelle chez la même espèce similaires à celles enregistrées dans la présente étude. Selon cet auteur, les mâles de $P$. leonensis sont matures à $27 \mathrm{~mm}$ et les femelles le sont à 28 mm (LS). Dans ce même lac, la pêche des Clupeidae s'effectue avec des sennes de plage de maille de 0,1 mm de côté (Yaro, 2003). Selon Duponchelle et Panfili (1998), la maturité sexuelle précoce ou le nanisme sont des adaptations écophysiologiques que peuvent présenter des poissons soumis à des pressions pour pérenniser leur espèce. Ainsi, la maturité sexuelle précoce observée dans ce travail serait due à la pression de pêche exercée sur le stock de $P$. leonensis dans le lac de Taabo.

La présence de $P$. leonensis femelles matures pendant toute l'année (sauf le mois de juillet) dans le lac de Taabo montre que $P$. leonensis se reproduit durant cette période. Cependant, l'évolution du rapport gonadosomatique couplée aux variations des 
pourcentages d'individus femelles matures montre que les plus grands nombres de poissons matures sont enregistrés en janvier, avril, juin et août et que les valeurs maximales du RGS sont atteintes au cours de ces mêmes périodes. Ces observations indiquent que la ponte est maximale pendant ces mois dans le lac de Taabo. Dans le lac de Kossou, les activités de reproduction de ce poisson se situe entre janvier et juillet (Koné et al., 2011). Dans le lac de Taabo, l'effort de pêche fourni pour la capture de $P$. leonensis est important durant toute l'année (au moins 246 sorties de pêche/mois et en moyenne 295 sorties/mois). Cet effort fourni constitue par ailleurs un important prélèvement de poissons par la pêche. En d'autres termes, la pêche commerciale soustrait des plans d'eau des géniteurs en pleine activité reproductrice, ce qui peut constituer une grave menace pour le renouvellement des stocks.

La distribution des fréquences de taille des ovocytes dans les gonades à différents stades de maturation montre que la ponte est fractionnée chez $P$. leonensis. Des ovocytes de petite taille constituant le stock de réserve sont présents à chaque stade de développement sexuel. Dans cette stratégie de ponte, des ovocytes immatures entrent en vitellogenèse pendant toute la durée de la période de ponte (N'Goran, 1995). Ce phénomène a déjà été observé chez d'autres poissons téléostéens par Rinchard et al. (1998). Pour Déniel et al. (1989), la gamétogenèse redémarre à partir d'un stock d'ovocytes pas encore en vitellogenèse. Les ovocytes n'évolueraient qu'au cours du cycle sexuel suivant. Selon Otémé (2001), les ovaires présentent à un moment donné, un lot assez uniforme de gros ovocytes, en plus des ovocytes de taille moyenne faisant la transition avec un lot plus hétérogène d'ovocytes de petite taille à partir duquel se fait le recrutement des gros ovocytes. Ces observations indiquent que les cellules sexuelles subissent dans les ovaires, un développement de type asynchrone ou une série de maturation de type groupe synchrone.

\section{Conclusion}

Cette étude a permis de montrer que la pêche du Clupeidae Pellonula leonensis est intense dans la localité de Taabo. L'effort de pêche fourni pour la capture de ce poisson est de 3547 sorties de pêche/an et sa production annuelle est de $211860 \mathrm{~kg}$. Le sex-ratio est en faveur des mâles $(1: 0,68)$ et la reproduction s'effectue principalement de décembre à avril avec un important pic en janvier et un pic moins important en avril. Elle se poursuit accessoirement pendant les mois de juin et août.

La taille de première maturité sexuelle est de $27,45 \mathrm{~mm}$ pour les femelles et 27,48 mm (LS) pour les mâles. Le stock de $P$. leonensis subit une pression importante dans le lac de Taabo du fait de la pêche intensive couplée à l'utilisation des sennes de rivage entrainant la réduction de la taille des poissons. Des dispositions urgentes et idoines, relatives à la gestion rationnelle du stock de $P$. leonensis, s'imposent. Il est important dans un premier temps, de sensibiliser les populations sur les conséquences de l'utilisation des sennes de plage à petites mailles sur les stocks de $P$. leonensis. Il serait ensuite souhaitable d'interdire l'usage des sennes de plage pour la pêche de ce poisson sur ce lac surtout pendant la période de reproduction maximale (décembre à avril). L'application de cette interdiction devra être rigoureusement suivie par des contrôles effectués par des agents des Eaux et Forêts. Ces mesures permettront ainsi d'avoir des poissons de taille normale et l'exploitation halieutique pourra perdurer.

\section{REMERCIEMENTS}

Nous remercions l'Etat de Côte d'Ivoire et le Centre Suisse de Recherche Scientifique (CSRS) pour le financement de cette étude à travers le Programme d'Appui Stratégique à la Recherche Scientifique 
(PASRES). Nous témoignons notre reconnaissance à toutes les personnes qui ont contribué à la réussite de ce travail.

\section{REFERENCES}

Aliko NG, Da Costa KS, Konan KF, Ouattara A, Gourène G. 2010. Fish diversity along the longitudinal gradient in a manmade lake of West Africa, Taabo hydroelectric reservoir, Ivory Coast. Ribarstvo, 68(2): 47-60.

Atsé BC, Konan KJ, Kouassi NJ. 2009. Biologie de la reproduction du Cichlidae Tylochromis jentinki dans la lagune Ebrié (Côte d'Ivoire). Cybium, 33(1): 1119.

Chemmam-Abdelkader B, Ezzeddine-Najaï S, Kraiem MM. 2007. Période de ponte, sex-ratio et maturité sexuelle du brochet de mer Sphyraena sphyraena (Teleostei, Sphyraenidae) au nord et à l'est de la côte tunisienne (Méditerranée occidentale et centrale). Bull. Inst. Natl. Sci. Tech. Oceanogr. Peche Salammbo, 34: 5 -10.

Da Costa KS, Dietoa YM. 2007. Typologie de la pêche sur le lac Faé (Côte d'Ivoire) et implications pour une gestion rationnelle des ressources halieutiques. Bull. Fr. Pêche Piscic., 384 : 1-14.

Déniel C, Le Blanc C, Rodriguez A. 1989. Comparative study of sexual cycles oogenesis and spawning of two Soleidae Solea lascaris and S. impar on Western coast of Britany. J. Fish Biol., 35: 49-58.

Duponchelle F, Panfili J. 1998. Variations in age and size at maturity of female tilapia, Oreochromis niloticus, populations from man-made lakes of Côte d'Ivoire. Environ. Biol. Fish., 52: 453-465.

FAO. 2012. La Situation Mondiale des Pêches et de l'Aquaculture. FAO : Rome. 241 p.

Kingdom T, Allison ME. 2009. Aspects of the reproductive biology of Pellonula leonensis (Boulenger, 1916; Clupeidae) in the Lower Nun River, Niger Delta. Curr. Res. J. Biol. Sci., 1(3): 68-71.

Konan KJ. 2010. Etude des paramètres de populations, de la biologie de reproduction et des stratégies alimentaires du Cichlidae Tylochromis jentinki (Steindachner, 1895) dans la lagune Ebrié (Côte d'Ivoire). Thèse de Doctorat, Université de Cocody, Abidjan, p.176.

Koné N, Berté S, Kraidy ALB, Kouamélan EP, Koné T. 2011. Biologie de la reproduction du Clupeidae Pellonula leonensis Boulenger, 1916 dans le lac de barrage de Kossou (Côte d'Ivoire). J. Appl. Biosci., 41: 2797 - 2807.

Kouassi KL, Gone DL, Meledje NH, Wognin AV, Aka K. 2007. Hydrologie et évolution spatio-temporelle des charges solides en suspension dans le lac du barrage hydroélectrique de Taabo (Côte d'Ivoire). EJSR, 18(3) : 464-478.

Layachi M, Melhaoui M, Srour A, Randani M. 2007. Contribution à l'étude de la reproduction et de la croissance du Rouget-barbet de vase (Mullus barbatus L., 1758) de la zone littorale méditerranéenne de Nador (Maroc). Bulletin de l'Institut Scientifique, Section Sciences de la Vie, 29: 43-51.

Lévêque C, Paugy D. 2006. Caractéristiques générales de la faune ichtyologique. In Les poissons des Eaux Continentales Africaines: Diversité, Ecologie, Utilisation par l'Homme, Lévêque C, Paugy D. (eds). Editions de l'IRD: Paris; 45-57.

Lévêque C, Paugy D, Duponchelle F. 2006. La reproduction. In Les Poissons des Eaux Continentales Africaines: Diversité, Ecologie, Utilisation par l'Homme, Lévêque C, Paugy D. (eds). Editions de l'IRD: Paris; 148176.

Mehanna SF. 2007. Stock assessment and management of the Egyptian Sole Solea 
aegyptiaca Chabanaud, 1927 (Osteichthyes: Soleidae), in the Southeastern Mediterranean, Egypt. Turk. J. Zool., 31: 379-388.

N'Goran YN. 1995. Biologie, écologie et pêche de l'ethmalose: Ethmalosa fimbriata (Bowdich, 1825) en lagune Aby (Côte d'Ivoire). Thèse de Doctorat, Université de Bretagne Occidentale, p. 195.

Otémé ZJ. 2001. Contribution à l'étude de la physiologie de la reproduction du silure Heterobranchus longifilis Valenciennes, 1840 (Clariidae): Gamétogenèses naturelle et induite. Thèse de Doctorat

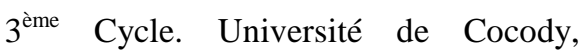
Abidjan, p.149.

Otobo FO. 1978. The reproduction biology of Pellonula afzeliusi Johnels, and Sierrathrissa leonensis Thys Audenaerde in Lake Kainji, Nigeria. Hydrobiologia, 61(2): $99-112$.

Paugy D. 2002. Reproductive strategies of fishes in a tropical temporary stream of the Upper Senegal basin: Baoule River in Mali. Aquat. Living Resour., 15(1): 25-35.

Rinchard J, Poncin R, Kestemont P. 1998. Croissance ovocytaire et régulation stéroïdienne chez les poissons à pontes unique et multiples: une revue. Annls Limnol., 34(2): 211-225.

Santos JNS, Araùjo FG, Silva MA, Vasconcellos RM. 2007. Sex-ratio and sexual dimorphism of the anchovy Anchoa januaria (Actinipterygii,
Engraulidae) in a tropical bay in southeastern Brazil. J. Fish Biol., 71: 877-888.

Savané I, Konaré A. 2010. Le climat. In Atlas de la Biodiversité de l'Afrique de l'Ouest (Tome III), Konaté S, Kampmann D (eds.). Côte d'Ivoire. Abidjan et Frankfurt/Main; 124-125.

Silva TCG, Cavalcante DP, Queiroz HL. 2013. Aspects of the reproductive biology of Aequidens tetramerus (Cichilidae) in Várzea of the Middle Solimões River Brazilian Amazon. Uakari, 9(2): 63 - 74.

Sylla S, Atsé BC, Kouassi NJ. 2009. Stratégie de Reproduction du Carangidae Trachinotus teraia Cuvier, 1832 dans la lagune Ebrié (Côte d'Ivoire). Sciences \& Nature, 6(1): 83 - 94

Vanga AF. 2011. Evolution de la pêche au lac d'Ayamé depuis l'expulsion des pêcheurs non nationaux (Côte d'Ivoire). Tropicultura, 29(1): 8-13.

Yao AA. 2008. Données préliminaires sur la pêcherie du petit pélagique Pellonula leonensis Boulenger, 1916 (Pisces; Clupeidae) dans le lac de barrage de Taabo (Bassin du Bandama, Côte d'Ivoire). Mémoire de D.A.A., ESA/INP-HB/CNRA, Yamoussoukro, $87 \mathrm{p}$.

Yaro I. 2003. An overview of the clupeid fishing gear of kainji Lake. In Proceedings of the $16^{\text {th }}$ Annual Conference of the Fisheries Society of Nigeria, Eyo AA, Ajao EA (eds). Mainduguri, 132-136. 